\title{
Characterization of Adaptive Optics at Keck Observatory
}

\author{
M. A. van Dam, B. A. Macintosh
}

This article was submitted to SPIE Annual Meeting, San Diego, California

08/03/2003 - 08/08/2003

U.S. Department of Energy

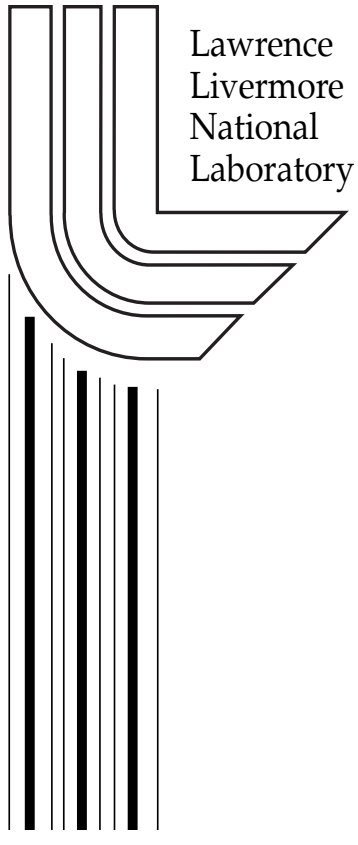

July 24, 2003 
This document was prepared as an account of work sponsored by an agency of the United States Government. Neither the United States Government nor the University of California nor any of their employees, makes any warranty, express or implied, or assumes any legal liability or responsibility for the accuracy, completeness, or usefulness of any information, apparatus, product, or process disclosed, or represents that its use would not infringe privately owned rights. Reference herein to any specific commercial product, process, or service by trade name, trademark, manufacturer, or otherwise, does not necessarily constitute or imply its endorsement, recommendation, or favoring by the United States Government or the University of California. The views and opinions of authors expressed herein do not necessarily state or reflect those of the United States Government or the University of California, and shall not be used for advertising or product endorsement purposes. 


\title{
Characterization of adaptive optics at Keck Observatory
}

\author{
Marcos A. van Dam and Bruce A. Macintosh \\ Lawrence Livermore National Laboratory, \\ P. O. Box 808, Livermore, CA 94550
}

\begin{abstract}
In this paper, the adaptive optics (AO) system at Keck Observatory is characterized. The AO system is described in detail. The physical parameters of the lenslets, CCD and deformable mirror, the calibration procedures and the signal processing algorithms are explained. Results of sky performance tests are presented: the AO system is shown to deliver images with an average Strehl ratio of up to 0.37 at $1.59 \mu \mathrm{m}$ using a bright guide star. An error budget that is consistent with the observed image quality is presented.
\end{abstract}

Keywords: Adaptive optics, wavefront sensing, Shack-Hartmann, Keck Observatory

\section{INTRODUCTION}

Adaptive optics (AO) has found widespread use in astronomical settings to compensate for atmospheric turbulence and telescope aberrations. ${ }^{1,2}$ At the W. M. Keck Observatory, there are identical adaptive optics systems on the Keck I and Keck II telescopes. Keck I is used only for interferometry and hence does not have a science camera, while Keck II supports a number of instruments. This paper attempts to characterize the performance of the Keck II adaptive optics system when used with the NIRC2 (near infrared) camera in imaging mode.

Characterization of $\mathrm{AO}$ systems has been undertaken at other observatories. ${ }^{3-5}$ It is important to understand the performance of the $\mathrm{AO}$ system under different atmospheric conditions and guide star brightnesses in order to predict the science output of an observation. It is also a good exercise to perform in conjunction with an AO optimization effort: knowledge of the error terms leads naturally to their elimination or mitigation. Finally, understanding the performance of current $\mathrm{AO}$ systems will lead to better design and implementation of future systems.

Extensive documentation on this characterization effort is posted on the Keck AO characterization website. ${ }^{6}$

\section{KECK ADAPTIVE OPTICS SYSTEM}

In this section, the components of the Keck AO system are described and analyzed. In particular, factors that affect performance are emphasized.

The Keck AO system consists of a tip/tilt mirror, a 349-actuator Xinetics deformable mirror (DM) and a dichroic beamsplitter that directs the visible light to the $20 \times 20$ subaperture Shack-Hartmann wave-front sensor and the infrared light to the science camera.

The science camera is a $1024 \times 1024$ pixel infrared camera with numerous filters. The camera has a subarraying capability that can be used to reduce the exposure time to as short as $8 \mathrm{~ms}$ with three seconds between consecutive exposures. In addition to science camera images, there are two other forms of data at our disposal to characterize the AO system. Telemetry consists of any data used by the AO system and is streamed at a rate of about $5 \mathrm{~Hz}$. This is useful for detecting trends over long periods of time. Telemetry is used by the AO system for many operations, such as obtaining the sky background or calculating the frame rate at which the AO system should operate. Diagnostics consist of 1000 consecutive samples of most values calculated by the wave-front controller, such as the centroids and the voltage applied to the DM or tip/tilt mirror. Writing the

Further author information: send correspondence to M.v.D, mvandam@igpp.ucllnl.org 
diagnostics to disk typically takes about 10 seconds a cycle, depending on how much data is stored. Taking diagnostics pauses the telemetry, so diagnostics are triggered sparingly.

There is also an atmospheric characterization tool that estimates Fried's parameter, $r_{0}$, from open- or closed-loop telemetry or diagnostics online. ${ }^{7}$

\subsection{Lenslets}

The lenslets are $20 \times 20$ acrylic lenslets from AOA with a pitch of $200 \mu \mathrm{m}$. There are three sets of lenslet arrays available, with focal lengths of $2.0,5.0$ and $7.9 \mathrm{~mm}$, corresponding to plate scales of $2.44,0.98$ and 0.62 arcsec/pixel respectively. Usually, the 2.44 arcsec plate scale lenslets are used. This is because the angular extent of the spot size is greater and hence does not change very much in the presence of turbulence. Also, essentially all the light is detected by the quad cells and does not leak out. However, the larger the angular extent of the spot, the higher the noise on the wave-front slope estimates. Although the centroid error is independent of spot size, this error is converted to a slope by dividing by a constant inversely proportional to the spot size. Hence, when the guide star is faint and the seeing is relatively good, the 0.98 arcsec plate scale is preferred. The 0.62 arcsec plate scale is never used.

When the DM is nominally flat, the RMS centroid measurements are 0.108 centroids or 0.068 arcsec on the 2.44 arcsec plate scale and 0.13 centroids or 0.028 acrsec on the 0.98 arcsec plate scale. These centroid errors are randomly distributed and are believed to stem from the lenslets themselves. As a result, the wave-front sensor operates off null. The penalties paid for operating off null are an increase in noise because we are no longer operating in the steepest part of the transfer curve and dynamic calibration errors explained in Sect. 4.4 due to the changing spot size.

The lenslets are mapped to the corner of four neighboring actuators in what is commonly called the Fried configuration. ${ }^{8}$

\subsection{CCD}

The wave-front sensing CCD is a Lincoln Labs MIT $64 \times 64$ CCD. The readout electronics consists of four amplifiers, one for each of four $64 \times 16$ strips. There are $3 \times 3$ pixels for each subaperture, consisting of a $2 \times 2$ pixel quad cell with a guard band between adjacent subapertures.

In order to compute the error in the centroid estimate, some physical parameters of the CCD need to be determined.

\subsubsection{ADU per electron}

The CCD intensities are not measured in photons but in analogue-to-digital units (ADUs). The probability distribution of the number of photons detected by the CCD obeys Poisson statistics, with the variance being equal to the expected number of photons. This property is used to compute the electrons per ADU conversion factor. Wave-front sensing images were captured at the highest frame rate with different neutral density filters. First, each frame was normalized to remove overall intensity fluctuations within frames with the same filter. It can be assumed that the variation from frame to frame is due to intensity fluctuations in the light source rather than the fluctuations in photon detection. Then the images were flat-fielded. The slope of the graph of the variance of the pixel intensities versus the mean pixel intensity gives the inverse of the electrons per ADU factor, which takes a value of 1.99 .

\subsubsection{Dark current}

To find the dark current, dark frames were captured at different frame rates. The mean intensity was recorded and plotted as a function of exposure time. The slope of the graph gives the dark current, 4470 electrons/pixel/s. Dark current is strongly dependent on temperature and the CCDs are Peltier cooled to $-6^{\circ} \mathrm{C}$. The measured dark current is consistent with its theoretical value:

$$
I_{\text {dark }}=A T^{3 / 2} \exp \left[-E_{g} / 2 k T\right],
$$

where $I_{\text {dark }}$ is the dark current, $E_{g}=1.2 \mathrm{eV}$ is the silicon band gap energy, $T$ is the temperature in Kelvin, $k$ is Boltzmann's constant and $A$ is a proportionality constant equal to $2.15 \times 10^{8}$ according to Lincoln Labs. 


\subsubsection{Read noise}

For a dark frame at $672 \mathrm{~Hz}$, the dominant signal is read noise. Removing the contribution from the dark current, we obtain a read noise standard deviation of 6.5 electrons.

\subsubsection{Spot size}

The size of the wave-front sensing spot was found by scanning the artificial light source across the focal plane and measuring the centroids. ${ }^{7}$ The full-width-half-maximum (FWHM) of a Gaussian spot is related to the maximum slope, $m$, of the "displacement in arcsec versus centroid" plot by

$$
\mathrm{FWHM}=\frac{2 \times 2.355}{\sqrt{2 \pi} m} .
$$

The spot sizes (FWHM) were found to be $1.30,0.52$ and 0.40 for the $2.44,0.98$ and 0.62 arcsec plate scales respectively. This compares with a diffraction-limited spot size of 0.23 at $700 \mathrm{~nm}$. The discrepancy is well explained by postulating that the measured intensity is a convolution of a diffraction-limited spot and a Gaussian with a FWHM of 0.50 pixels. The blurring of the spot is almost certainly due to charge diffusion and the extent of the charge diffusion is consistent with other measurements of similar CCDs. ${ }^{9}$ The effect of the charge diffusion on the spot size increases with increasing plate scale.

\subsubsection{Linearity}

The CCD has two amplifier gains. The most commonly used mode has a gain of 1.99 electrons per ADU. The other mode, which has a gain of 4.00 electrons per ADU, is only used on the brightest stars. Saturation of the wave-front sensor CCD occurs at 2000 ADU per pixel, corresponding to a guide star of magnitude 4.5. For brighter stars, a neutral density filter may be used to limit the light on the wave-front sensor.

The third of the four CCD amplifiers exhibits non-linear behavior. The ADU/electron ratio for that CCD strip increases gradually from about $60 \%$ of its maximum value in the limit where there is no light, to almost $100 \%$ at about 150 counts per ADU. This presents two problems: First, the read noise in that strip in ADU is the same as for the other strips. Hence, the read noise in electrons at low light levels is almost double. The dark current, in electrons, remains the same. Second, one column of subapertures is straddled by two strips with different amplifiers. Two of the pixels corresponding to one subaperture are located in one strip and the other two pixels in another strip. Hence, the centroid reading in the absence of any wave-front aberration is light dependent for stars fainter than magnitude 10. The temporary solution has been to calibrate the system at different light levels. In the near future, we intend to shift the CCD by one pixel so that no subapertures are straddled by the third strip and another strip.

\subsection{Signal processing}

\subsubsection{Reconstruction matrix}

The fact that the Keck telescope is on an alt/az mount with the adaptive optics system on a Nasmyth platform means that as the telescope tracks a star, the hexagonal pupil image rotates on the wavefront sensor camera and on the DM. The Keck telescope has an image derotator to compensate for the image rotation, so the pupil rotates as the telescope tracks an object. Because the pupil is not circular, but a serrated hexagon, the illuminated subapertures change with time. At any given time, 240 out of the 304 subapertures are active and used to reconstruct the wave-front. The other subapertures are discarded as they have little or no illumination. Every time the rotator changes position by more than one degree, the calculation of a new reconstruction matrix is triggered. Until very recently, the matrix inversion was performed using an SVD algorithm. ${ }^{10}$ This has been replaced by a Bayesian reconstructor that uses the statistics of Kolmogorov turbulence and the relative number of ADUs per subaperture as prior information. There is a parameter that can be adjusted depending on the signal-to-noise ratio. This would be the optimal reconstructor in the open-loop case; simulations and extensive sky testing have demonstrated that it also performs very well in closed loop. An improvement in image quality corresponding to a reduction in quadrature of the RMS wave-front error of about $100 \mathrm{~nm}$ for bright guide stars, a figure that increases as for fainter guide stars. The most visible improvement is the elimination of the four spots in a square pattern in the image due to waffle on the DM. 


\subsubsection{Frame rate and delay}

The wave-front controller can operate at frame rates in the range of 55 to $672 \mathrm{~Hz}$. The upper limit is set by the speed at which the computers can multiply the reconstructor matrix by the centroid measurements. There are separate control loops for the tip/tilt mirror and the DM. The compute delay times, from the time that the CCD is read until the time that the tip/tilt mirror and DM voltages are updated, are $1.65 \mathrm{~ms}$ and $2.2 \mathrm{~ms}$ respectively.

\subsubsection{Loop gains and compensator}

Each of the control loops has its own controller. The tip/tilt loop is a straight integrator with a variable loop gain, $k_{\mathrm{t}}$. Its transfer function can be written as

$$
H_{\mathrm{t}}(z)=\frac{k_{\mathrm{t}} z}{z-1},
$$

where $z$ is the complex Z-transform variable. The DM loop has a double pole compensator that can be expressed as

$$
H_{\mathrm{d}}(z)=\frac{k_{\mathrm{d}} z^{2}}{(z-l)(z+w)} .
$$

The compensator weight, $w$, is set to 0.25 , a value designed to increase the bandwidth of the controller. In addition, there is a leaky integrator with the leak factor, $l$, set to 0.99 , to ensure that invisible modes do not build up in the DM. The loop gain, $k_{\mathrm{d}}$, is also variable.

The optimum loop gains depend on the turbulence strength and speed, the extent and brightness of the guide star and the frame rate. In routine operation, the loops gains and the frame rate are set using a look-up table that has the median number of ADUs per subaperture per second as its only input. We plan to optimize the gains online using centroid telemetry.

\subsection{Deformable mirror}

The Keck AO system consists of a Xinetics 349-actuator deformable mirror (DM). Oppenheimer et al. investigated the influence function of this mirror. ${ }^{11}$ The influence function describes the optical effect of the deformation of the mirror when one volt is applied to the pre-amplifier. The influence function, $s(x, y)$, can be very well approximated using the difference of two Gaussians:

$$
s(x, y)=\left(\frac{w_{1}}{2 \pi \sigma_{1}^{2}} \exp \left[\frac{-\left(x^{2}+y^{2}\right)}{2 \sigma_{1}^{2}}\right]+\frac{w_{2}}{2 \pi \sigma_{2}^{2}} \exp \left[\frac{-\left(x^{2}+y^{2}\right)}{2 \sigma_{2}^{2}}\right]\right) \times 470 \mathrm{~nm} .
$$

The values for the constants are: $w_{1}=2, w_{2}=-1, \sigma_{1}=0.54$ subapertures and $\sigma_{2}=0.85$ subapertures.

\subsection{Calibration}

Calibrating an AO system well is crucial to obtain good performance on the sky. Here, a brief description of the most important calibration procedures is presented. The calibrations are performed using a white light source that is $10 \mu \mathrm{m}$ in diameter and located at the focus of the telescope.

\subsubsection{DM to lenslet registration}

It is important to have the correct registration between the DM and the lenslet. Otherwise, the waffle mode, where actuators are lined up in a checkerboard pattern, is observed on the DM when the loops are closed. The algorithm used to perform the DM-to-lenslet registration is adapted from a paper by Oliker. ${ }^{12}$ It involves putting waffle on the DM and ensuring that the wave-front sensor does not sense any signal.

\subsubsection{System matrix generation}

The system matrix relates the motion of each actuator to the centroids produced at the wave-front sensor. It is calculated by moving each actuator by $\pm 0.2 \mu \mathrm{m}$ and recording the difference in the centroids. Centroids corresponding to subapertures that are more than two subapertures away from the actuator are set to zero to reduce the level of noise. The actuator motion is chosen such that it yields a good signal-to-noise ratio in the centroid measurements while ensuring that the the wave-front sensor is operating in its linear range. 


\subsubsection{Image sharpening}

There are aberrations on the imaging leg that are not sensed by the WFS and vice versa. In the absence of any external aberrations, one would want the DM to have the shape that maximizes the Strehl ratio. Image sharpening refers to the process of finding this optimum shape of the DM. The non-common path aberrations are calibrated as follows. First, the DM is flattened using a WYKO interferometer: The phase on the DM is measured with the interferometer and the voltage applied is altered iteratively so as to cancel the measured phase. Unfortunately, the interferometer does not see some of the actuators at the edges and these cannot be flattened properly. Next, the phase diversity algorithm of Loefdahl and Scharmer ${ }^{13}$ is employed to remove the Zernike polynomials ${ }^{14}$ up to spherical aberration. Typically, $100 \mathrm{~nm}$ of RMS wave-front are applied to the DM in order to correct for these non-common-path aberrations. The algorithm reduces the wavefront error seen on the artifical source from $150 \mathrm{~nm}$ to $113 \mathrm{~nm}$. These numbers take into account the finite size of the source (10 $\mu \mathrm{m}$ in diameter).

Once the optimum shape is found, it is placed on the DM and the centroids measured by the wave-front sensor are defined to be the centroid origins. In closed-loop operation, the DM and TT mirror are driven in such a way to nullify the difference between the centroids and the centroid origins. Hence, upon immediately closing the loops, neither the tip/tilt mirror nor the DM should move.

The problem with calibrating by offsetting the centroids when the centroids are measured with a quad cell is that the size of the spot on the CCD increases on the sky due to the effect of atmospheric turbulence. The centroid measurement on a quad cell is inversely proportional to the spot size. As the spot size increases, the $\mathrm{AO}$ system overcompensates for the non-common path aberrations, thereby introducing a wave-front error. A simple partial solution involves measuring the spot size on the sky to see how much it has changed. This is done by offsetting all the centroid origins by a constant value while the AO loops are closed on a guide star and measuring the displacement of the image on the science camera. Since the pixel extent in arcseconds of the science camera is known, one can find the rate of change of centroids as a function of image motion. The spot size can be recovered using Eq. (2). Under good atmospheric conditions $\left(r_{0}=20 \mathrm{~cm}\right)$, the spot increases in size from 1.3" to 1.6 ". Consequently, the centroid origins are routinely scaled by 0.8 . Unfortunately, the spot size is constantly changing as the seeing changes. In addition, if the guide star is an extended source, the spot size will be even greater. It is intended that in the near future, the spot size will be monitored in real time using telemetry readings of the DM voltages using a technique suggested by Véran and Herriot. ${ }^{15}$

\section{EXPERIMENTAL RESULTS}

Images of a number of stars under a variety of seeing conditions were captured between May 22 and June 16, 2003. A figure of merit often used to characterize the error of an AO system is the Strehl ratio, $S$. It is defined as the ratio of the maximum value of the measured point-spread function over the maximum of the diffraction-limited point spread function. The Strehl ratios of all the images were calculated using a window with a diameter of 2.4 arcsec. Figure 1 plots the Strehl at with the $\mathrm{H}$ continuum $(1.59 \mu \mathrm{m})$ filter as a function of guide star brightness and the corresponding RMS wave-front error from the Maréchal approximation. Only the results corresponding to the optimal frame rate and lenslet array plate scale are displayed, as these are the settings that are used in practice.

The magnitude 13 stars were imaged at K' and hence are not included in the Strehl plot. The images had exposure times of between three and 15 seconds.

The FWHM of the best corrected images is 36.5 mas, while the best images on the magnitude 12 star had a FWHM of 40 mas. By comparison, the diffraction-limited FWHM is 33.6 mas.

\section{BRIGHT STAR ERROR BUDGET}

The Strehl ratio is related to the wave-front errors via the Maréchal approximation, ${ }^{2}$

$$
S=\exp \left[-\sigma_{\phi}^{2}\right] \exp \left[-\sigma_{\chi}^{2}\right]
$$



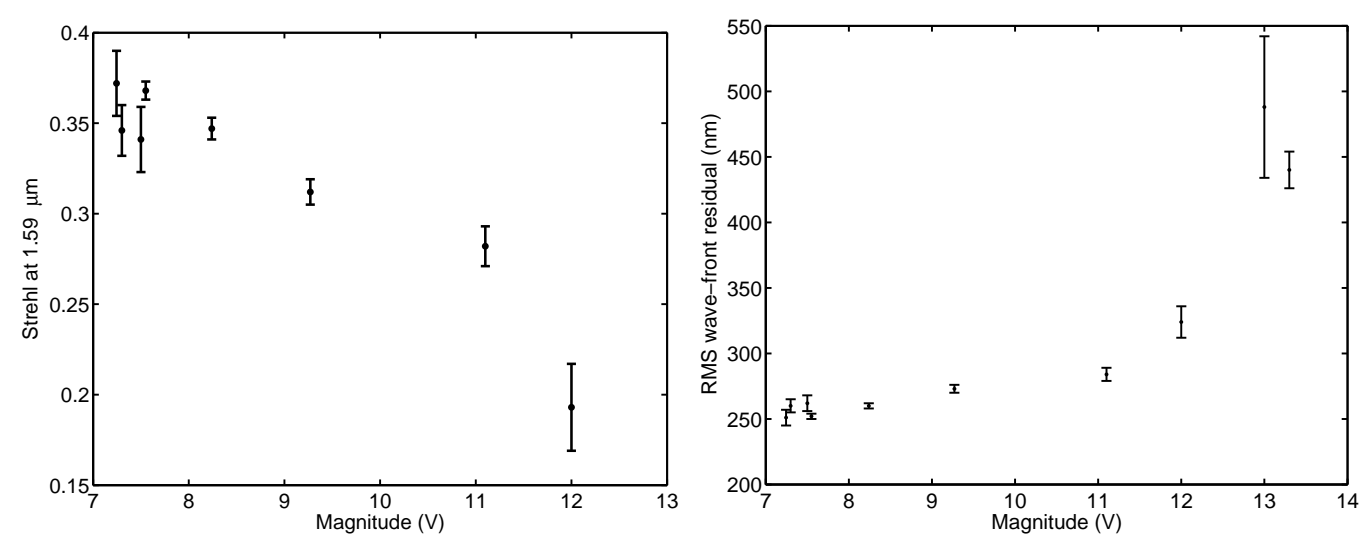

Figure 1. Strehl ratio at $1.59 \mu \mathrm{m}$ (left) and the RMS wave-front error (right). The points represent the mean of about 10 images and the error bars represent the $1 \sigma$ spread about the mean.
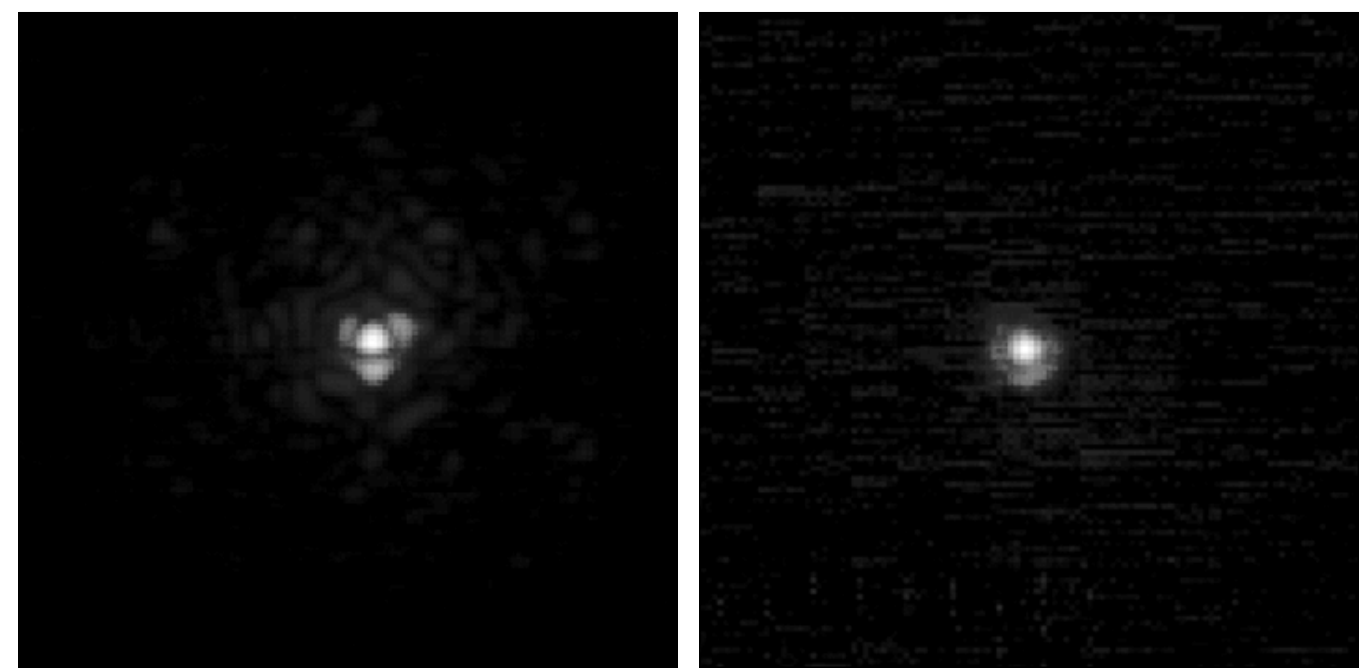

Figure 2. Best images obtained on a bright star (left) and on a $12^{\text {th }}$ magnitude star (right). The pixels are 10 mas across.

where $\sigma_{\phi}^{2}$ is the wave-front phase variance and $\sigma_{\chi}^{2}$ is the variance of the log-normal amplitude at the pupil plane. $\mathrm{An} \mathrm{AO}$ system with a single wave-front corrector conjugate the ground can only correct the wave-front phase aberrations. Hence the goal of the AO system is to minimize the wave-front error. In this section, the individual wave-front error terms are presented. The phase error is inversely proportional to the wavelength, so the Strehl ratio increases with increasing wavelength. In the engineering tests, the filter used was $\mathrm{H}$ continuum, a narrow band filter centered at $1.59 \mu \mathrm{m}$ with a $1 \%$ passband. The reason for this choice is that a narrowband filter was required in order to avoid saturation of the science camera when using bright targets. For the same reason, guide stars brighter than magnitude six are not used for engineering, although they can be used for science with the insertion of neutral density filters. A short wavelength enables one to more easily discern performance changes in real time, as the image quality varies more than for the longer wavelengths. All Strehl ratios quoted in this paper correspond to $1.59 \mu \mathrm{m}$. Strehl ratios at other wavelengths can be estimated using Eq. (6). All the wavefront errors are root-mean-squared (RMS) errors.

It is important to emphasize that the individual error terms are assumed to be uncorrelated and are added in quadrature (i.e., their variances are added). This means that small errors have a negligible effect on the total error budget in the presence of much larger terms. 
In calculating the error terms, the atmospheric turbulence is assumed to be Kolmogorov, ${ }^{16}$ with a turbulence strength defined by $r_{0} .{ }^{17}$ Tests have shown that the Kolmogorov model of atmospheric turbulence describes the wave-front aberrations encountered at Keck Observatory well, with the exception of tip/tilt aberrations, which are augmented by telescope vibrations. ${ }^{7}$ Hence, the measurements of $r_{0}$ are made from tip/tilt-removed images or diagnostics.

Many images and diagnostics were taken on June 15, 2003. From the images, the RMS wave-front error was estimated to be $260 \mathrm{~nm}$. It was estimated that $r_{0}$ at $500 \mathrm{~nm}$ was $20 \mathrm{~cm}$.

\subsection{Camera errors}

The Strehl ratio measured on the artificial light source after image sharpening is 0.77 , corresponding to $130 \mathrm{~nm}$ of wave-front error. However, the extent of the light source, 13.8 milliarcseconds (mas), reduces the Strehl by the same amount as a $64 \mathrm{~nm}$ RMS wavefront aberration. Hence, the actual wave-front error is $113 \mathrm{~nm}$. Most of the residual error consists of high-order aberrations as discussed in Sect. 2.5.3. Figure 4.1 shows the phase error after image sharpening computed using the Gerchberg-Saxton phase retrieval algorithm. ${ }^{18}$ Because of the symmetry of the aperture, there is an ambiguity about the sign and the orientation of the phase. ${ }^{19}$

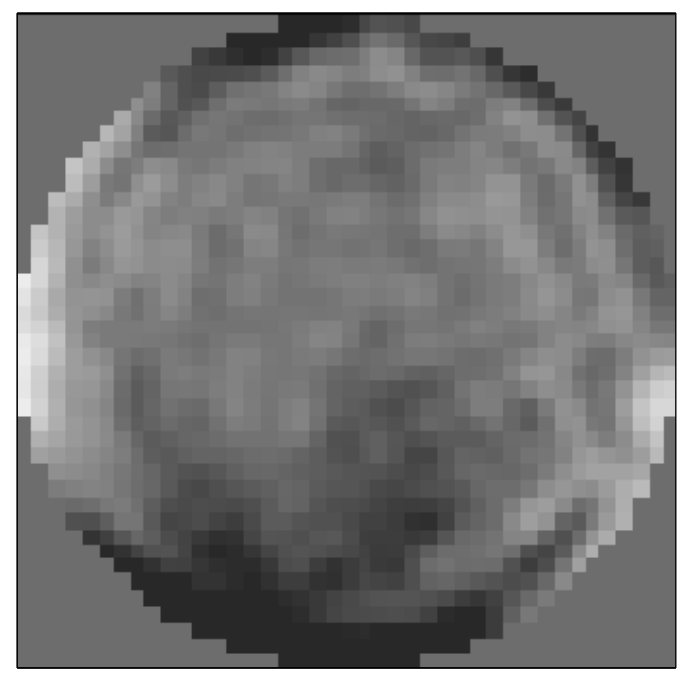

Figure 3. Phase map of the residual error after image sharpening.

\subsection{Fitting error}

The fitting error is defined to be the component of the wave-front that cannot be corrected by the deformable mirror. This error depends on the spacing between of actuators, the influence function of the actuators and the spatial power spectrum of the turbulence and the telescope errors. There are two sources of error that the DM has to correct for: the atmospheric turbulence and the telescope error.

Using the influence function described in Sect. 2.4, it was found by simulation that the RMS fitting error, $\sigma_{\text {fitting }}$, for Kolmogorov turbulence is equal to $33.2 r_{0}^{-5 / 6} \mathrm{~nm}$. The general form of the fitting error is given by ${ }^{2}$

$$
\sigma_{\text {fitting }}=\sqrt{a_{f}}\left(\frac{d}{r_{0}}\right)^{5 / 6} \frac{\lambda}{2 \pi},
$$

where $d$ is the spacing between the actuators, $\lambda$ is the wavelength at which $r_{0}$ is measured and $a_{f}$ is a constant that depends on the influence function. The value of $a_{f}$ calculated here is 0.46 , which is higher than the range of 0.28 to 0.34 presented in Hardy for a continuous-plate mirror. ${ }^{2}$ For $r_{0}=20 \mathrm{~cm}, \sigma_{\text {fitting }}=128 \mathrm{~nm}$. 
The Keck primary mirror consists of 36 hexagonal segments. Each segment has low-order aberrations and a dimple in the center resulting from the manufacturing process. In addition, there are stacking (piston) and pointing (tip/tilt) errors of each segment relative to its neighbors. It is estimated that after phasing the mirror segments, ${ }^{20}$ there are about $110 \mathrm{~nm}$ of wave-front residual error. The segments are not routinely phased before an $\mathrm{AO}$ run so this error could be higher. The fitting error on after correction by the DM was found to be 60 $\mathrm{nm}$ by simulating the errors on the primary mirror of the telescope: It is not possible to correct well for the segment discontinuities.

\subsection{Bandwidth error}

The bandwidth errors are due to the finite sampling rate of the atmospheric turbulence and the delay between the centroid measurements and the DM and tip/tilt command updates. Here, the errors are calculated by taking the power spectral density of the residual wave-front from the centroid diagnostics data, subtracting the noise floor and integrating the area under the curve. A more thorough analysis that takes into account the power spectral density of the noise will be presented in a future paper.

The power spectral density of the tip/tilt and the DM are plotted in Figure 4. The tip/tilt curve is averaged over several diagnostics with the same loop gain in order to reduce the random fluctuations and see the underlying trends. The power spectral densities are consistent with what was is expected by modeling the
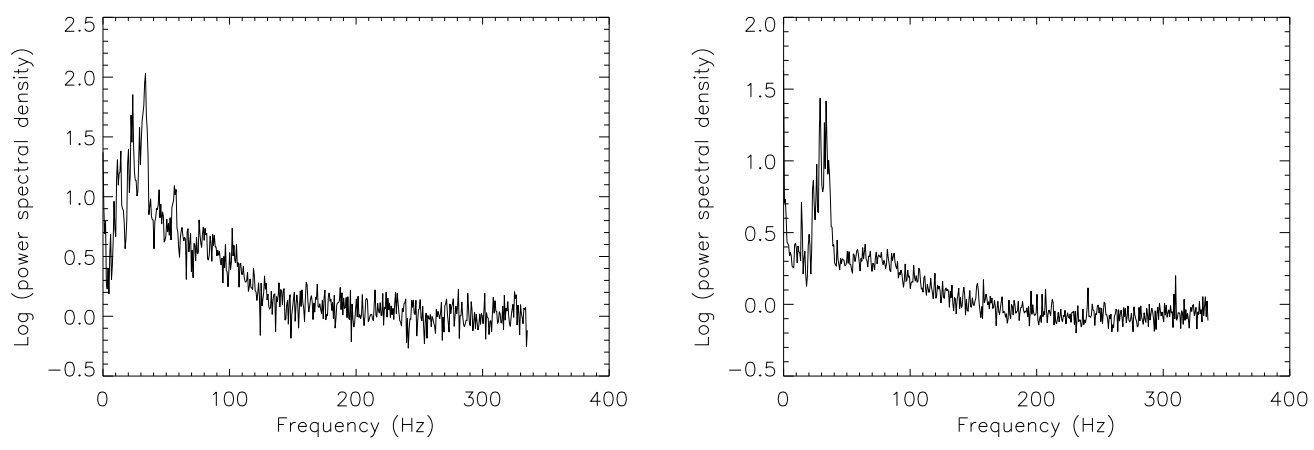

Figure 4. Logarithm of the power spectral density in $\mathrm{nm}^{2} / \mathrm{Hz}$ for the tip/tilt mirror (left) and the DM (right).

transfer function of the system. ${ }^{21}$ From the plots of the power spectra, it can be seen that there are vibration peaks at frequencies ranging from 20 to $40 \mathrm{~Hz}$ superimposed on Kolmogorov turbulence.

For the data of June 15, the average RMS bandwidth errors were $128 \pm 15 \mathrm{~nm}$ for the tip/tilt mirror and $55 \pm 7$ for the DM.

\subsection{Miscellaneous error}

There are several other error terms that have not yet been calculated but should be part of a complete error budget.

Due to the limited number of photons detected by the wave-front sensor, the centroid estimates are noisy. For a star brighter than magnitude seven, both the tip/tilt and the DM noise error terms are estimated to be less than $20 \mathrm{~nm}$ each.

The wave-front sensing and reconstruction restrict the performance of the DM. For example, while all the actuators were assumed to be independently controlled in the calculation of the fitting errors, in actual fact many of the actuators have no neighboring subapertures and are slaved to the average value of their neighboring actuators. Also, because the wave-front reconstructor has no knowledge of either the influence function of the mirror or the analogous response function of the wave-front sensor, even in the absence of noise, the actuators are not driven to their optimum values. In other words, even if the DM had an infinite number of degrees of freedom, there would be an error associated with the finite number of measurements. 
Because the wave-front sensor measures the average wave-front slope over the subaperture, any wave-front aberrations with a spatial frequency higher than the Nyquist criterion will be aliased to a lower spatial frequency, resulting in an error in the wave-front estimate. This term has a magnitude of about one third of the fitting error. $^{22}$

As stated in Sect. 4.2, the primary mirror segments are not phased before adaptive optics science nights; an estimate of the alignment of the segments is needed.

There are static calibration errors: The centroid offsets, influence matrix and the DM-to-lenslet registration all have some error associated with them. In addition, there are dynamic calibration errors. As the spot size increases due to the seeing, the offset centroids no longer correspond to the same wave-front slope for which they were calibrated. This is accounted for to some degree by scaling the centroid offsets, but the scaling is not exact as each spot is of a different size at any given time.

Finally, amplitude fluctuations in the pupil plane have the effect of reducing the Strehl ratio via Eq. (6). An estimate is needed of the scintillation, which depends on the height of the turbulence layers, for typical Mauna Kea atmospheric conditions.

\subsection{Summary of error terms}

To find all the total RMS wave-front error, the variances of all the individual terms are added in quadrature:

$$
\begin{aligned}
\sigma_{\text {total }} & =\left(\sigma_{\text {camera }}^{2}+\sigma_{\text {fitting }}^{2}+\sigma_{\text {telescope }}^{2}+\sigma_{\text {tt }-\mathrm{bw}}^{2}+\sigma_{\mathrm{dm}-\mathrm{bw}}^{2}\right)^{1 / 2} \\
& =\left(113^{2}+128^{2}+60^{2}+128^{2}+55^{2}\right)^{1 / 2} \\
& =228 \mathrm{~nm} .
\end{aligned}
$$

An additional $125 \mathrm{~nm}$ of error, comprised of all the smaller error terms, is needed to obtain the $260 \mathrm{~nm}$ of error estimated from the images.

\section{CONCLUSION}

The AO system at Keck Observatory has been characterized. The AO system is shown to deliver images with an average Strehl ratio of up to 0.37 at $1.59 \mu \mathrm{m}$ with bright guide star. This corresponds to an RMS wave-front error of $250 \mathrm{~nm}$. An error budget that is consistent with the observed image quality is presented. The major error terms on a bright guide star are the fitting error, the tip/tilt bandwith error and the internal calibration error, all of which are over $100 \mathrm{~nm}$ RMS. Of secondary importance are the DM bandwidth error and the telescope aberrations.

\section{ACKNOWLEDGMENTS}

This work was performed under the auspices of the US Department of Energy by the University of California, Lawrence Livermore National Laboratory, under contract W-7405-Eng-48. The work has been supported by the National Science Foundation Science and Technology Center for Adaptive Optics, managed by the University of California at Santa Cruz under cooperative agreement No. AST-9876783. The authors wish to thank the AO groups at both LLNL and Keck Observatory for their assistance. David Le Mignant (Keck Observatory) played a prominent role in this work but does not wish to be acknowledged as an author in protest at SPIE's involvement with the military.

\section{REFERENCES}

1. M. C. Roggemann and B. Welsh, Imaging through turbulence, CRC Press, Boca Raton, Fla. (1996).

2. J. W. Hardy, Adaptive Optics for Astronomical Telescopes, Oxford University Press, New York (1998).

3. F. Rigaut, D. Salmon, R. Arsenault, J. Thomas, O. Lai, D. Rouan, J. P. Véran, P. Gigan, D. Crampton, J. M. Fletcher, J. Stilburn, C. Boyer and P. Jagourel, "Performance of the Canada-France-Hawaii telescope adaptive optics Bonnette," PASP 110, 152-164 (1998). 
4. L. C. Roberts, Jr. and C. R. Neyman, "Characterization of the AEOS system," PASP 114, 1260-1266 (2002).

5. D. Gavel, E. Gates, C. Max, S. Olivier, B. Bauman, D. Pennington, B. Macintosh, J. Patience, C. Brown, P/ Danforth, R. Hurd, S. Severson, J. Lloyd, "Recent science and engineering results with the laser guidestar adaptive optics system at Lick Observatory," in Adaptive Optical System Technologies II, P. L. Wizinowich and D. Bonaccini, eds., Proc. SPIE 4839, 354-359 (2003).

6. M. A. van Dam and D. L. Le Mignant, "Keck adaptive optics characterization website," http://www.keck.hawaii.edu/realpublic/optics/aochar/index.html (2003).

7. M. Schoeck, D. Le Mignant, G. Chanan, P. L. Wizinowich and M. A. van Dam, "Atmospheric characterization with the Keck adaptive optics system I: open-loop data," Applied Optics 42, 3705-3720 (2003).

8. R. H. Hudgin, "Wave-front reconstruction for compensated imaging," J. Opt. Soc Am. 67, 375-378 (1977).

9. B. L. Ellerbroek, personal communication (2002).

10. P. J. Stomski, Jr. and J. C. Shelton, "Compensating for pupil rotation in the W. M. Keck Observatory adaptive optics system," in Adaptive Optical Systems Technology, P. L. Wizinowich, ed., Proc. SPIE 4007, 608-619 (2000).

11. B. R. Oppenheimer, D. Palmer, R. G. Dekany, A. Sivaramakrishnan, M. A. Ealey, T. R. Price, "Investigating a Xinetics Inc. deformable mirror," in Adaptive Optics and Applications, R. K. Tyson and R. Q. Fugate, eds., Proc. SPIE 3126, 569 (1997).

12. M. D. Oliker, "Alignment techniques for DM, lenslet, and WFS camera at the SOR," in Adaptive Optics and Applications, R. K. Tyson and R. Q. Fugate, eds., Proc. SPIE 3126, 595-604 (1997).

13. M. G. Loefdahl and G. B. Scharmer, "Wavefront sensing and image restoration from focused and defocused solar images," Astron. Astrophys. Suppl. Ser. 107, 243-264 (1994).

14. R. J. Noll, "Zernike polynomials and atmospheric turbulence," J. Opt. Soc. Am. 66, 207-211 (1976).

15. J-P. Véran and G. Herriot, "Centroiding gain compensation in Shack-Hartmann adaptive optics systems with natural or laser guide star," J. Opt. Soc. Am A 17, 1430-39 (2000).

16. F. Roddier, "The effect of atmospheric turbulence in optical astronomy", Progress in Optics, E. Wolf, ed. (North-Holland, Amsterdam, 1981), pp. 283-376.

17. D. L. Fried, "Optical resolution through a randomly inhomogeneous medium for very long and very short exposures", J. Opt. Soc. Am. 56, 1372-1379 (1966).

18. R. Gerchberg and W. Saxton "A practical algorithm for the determination of phase from image and diffraction plane picture", Optik 35, 237-246 (1972).

19. R. G. Lane, W. R. Fright and R. H. T. Bates, "Direct phase retrieval," IEEE Trans. Acoust., Speech, Signal Processing, ASSP-35, 520-526 (1987).

20. G. Chanan, C. Ohara and M. Troy, "Phasing the mirror segments of the Keck telecopes II: the narrow band phasing algorithm," Applied Optics 39, 4706-4714 (2000).

21. E. M. Johansson, D. S. Acton, J. R. An, K. Avicola, B. V. Beeman, J. M. Brase, C. J. Carrano, J. Gathright, D. T. Gavel, R. L. Hurd, O. Lai, W. Lupton, B. A. Macintosh, C. E. Max, S. S. Olivier, J. C. Shelton, P. J. Stomski, K. Tsubota, K. E. Waltjen, J. A. Watson and P. L. Wizinowich, "Initial performance of the Keck AO wavefront controller system," in Adaptive Optical Systems Technology, P. L. Wizinowich, ed., Proc. SPIE Vol. 4007, 600-607 (2000).

22. F. J. Rigaut, J.-P. Véran and O. Lai, "Analytical model for Shack-Hartmann-based adaptive optics systems", in Adaptive Optical System Technologies, D. Bonaccini and R. K. Tyson, eds., Proc. SPIE 3353 1038-1048 (1998). 